Research Paper

\title{
Efficacy of CapeOX plus Cetuximab Treatment as a First-Line Therapy for Patients with Extended RAS/BRAF/PIK3CA Wild-Type Advanced or Metastatic Colorectal Cancer
}

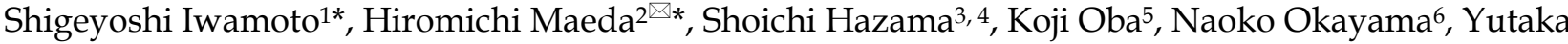 \\ Suehiro ${ }^{7}$, Takahiro Yamasaki ${ }^{7}$, Nobuaki Suzuki ${ }^{3}$, Hiroaki Nagano ${ }^{3}$, Junichi Sakamoto ${ }^{8}$, Hideyuki Mishima ${ }^{1}$, \\ Naoki Nagata9 \\ 1. Cancer Center, Aichi Medical University, Nagakute, Japan \\ 2. Cancer Treatment Center, Kochi Medical School Hospital, Kochi University, Nankoku, Japan \\ 3. Department of Gastroenterological, Breast and Endocrine Surgery, Yamaguchi University Graduate School of Medicine, Ube, Japan. \\ 4. Department of Translational Research and Developmental Therapeutics against Cancer, Yamaguchi University School of Medicine, Ube, Japan. \\ 5. Department of Biostatistics, Graduate School of Medicine, The University of Tokyo, Tokyo, Japan. \\ 6. Division of Laboratory, Yamaguchi University Hospital, Ube, Japan \\ 7. Department of Oncology and Laboratory Medicine, Yamaguchi University Graduate School of Medicine, Ube, Japan \\ 8. Tokai Central Hospital, Kakamigahara, Japan. \\ 9. Kitakyushu General Hospital, Kitakyushu, Japan. \\ *Equal contribution
}

$\triangle$ Corresponding author: Hiromichi Maeda, MD, PhD. Cancer Treatment Center, Kochi Medical School Hospital, Kochi University, Kohasu, Oko-cho, Nankoku, Kochi, 783-8505, Japan. E-mail; hmaeda@kochi-u.ac.jp, Telephone: +81-88-880-2370, Fax: +81-88-880-2371

(C) Ivyspring International Publisher. This is an open access article distributed under the terms of the Creative Commons Attribution (CC BY-NC) license (https://creativecommons.org/licenses/by-nc/4.0/). See http://ivyspring.com/terms for full terms and conditions.

Received: 2018.04.23; Accepted: 2018.07.25; Published: 2018.10.18

\begin{abstract}
Background: Oxaliplatin and capecitabine (CapeOX) combined with cetuximab is rarely used to treat advanced and metastatic colorectal cancer (mCRC). The present study aimed to clarify the clinical benefits of this treatment regimen when used as a first-line therapy in patients with expanded RAS/BRAF/PIK3CA wild-type mCRC, using the data and tumor specimens from two previously published Phase II clinical trials.

Methods: The gene mutation status and clinical data of 102 patients with KRAS wild-type mCRC, who received either of CapeOX + cetuximab or FOLFOX + cetuximab, were analyzed. The primary endpoint was response rate (RR) of CapeOX + cetuximab treatment in patients with extended RAS/BRAF/PIK3CA wild-type mCRC. RR comparisons and maximum tumor size changes between different treatment regimens and gene mutation status were set as key secondary endpoints.

Results: We identified 88 patients with extended RAS/BRAF/PIK3CA wild-type mCRC. Those treated with CapeOX + cetuximab $(n=52)$ had a $61.5 \%$ RR $(95 \% \mathrm{Cl}, 47.0-74.7 \%)$, while those treated with FOLFOX + cetuximab $(n=36)$ had a $66.7 \%$ RR $(95 \% \mathrm{Cl}, 49.0-81.4 \%)$. Patients with any mutation $(n=14)$ had a $42.9 \%$ RR $(95 \% \mathrm{Cl}, 17.1-71.1 \%)$. There were no significant differences between these three groups $(P=0.298)$. The disease control rate was $86.5 \%(95 \% \mathrm{Cl}, 74.2-94.4 \%)$ in the CapeOX + cetuximab group, and $88.9 \%(95 \% \mathrm{Cl}, 73.9-96.9 \%)$ in the FOLFOX + cetuximab group. Maximum tumor size changes were largest in patients with wild-type $\mathrm{mCRC}$ treated with FOLFOX + cetuximab followed by patients with wild-type mCRC treated with CapeOX + cetuximab, and then by those with any mutation $(-63.2 \%$, $-52.6 \%$, and $-27.3 \%$, respectively; $P=0.035$ ).

Conclusions: Patients with RAS/BRAF/PIK3CA wild-type mCRC had a sufficient RR following first-line treatment with CapeOX + cetuximab. These results suggest that this combination therapy should be considered as a treatment option for patients with advanced $\mathrm{mCRC}$.
\end{abstract}

Key words: cetuximab, BRAF, CapeOX, XELOX, PIK3CA, 


\section{Background}

The chemotherapeutic agent cetuximab binds to the extracellular domain of the epidermal growth factor receptor (EGFR), thereby inhibiting ligand binding and preventing signaling pathways required for cell proliferation, survival, and metastasis [1]. In the clinical setting, cetuximab improves the survival outcome of patients with metastatic colorectal cancers (mCRC) either in combination with cytotoxic agents or as a monotherapy [2-4]. However, cetuximab monotherapy provides no clinical benefits to mCRC patients with KRAS mutations [5]. BRAF, PIC3CA, and extended RAS mutations are also predictors of poor tumor response to cetuximab [6-8].

Cetuximab treatment is often provided with cytotoxic doublets. Combination therapy using oxaliplatin and orally administered capecitabine, known as CapeOX (or CAPOX), uses the central venous access port less frequently than combined 5-fluorouracil/leucovorin (5FU/LV) and oxaliplatin (FOLFOX) therapy [9]. This confers a significant advantage to patients as they are less likely to suffer catheter-related complications, such as infection, deep venous thrombosis, and catheter obstruction. Due to the comparable efficacy of CapeOX to FOLFOX treatment [10], the CapeOX regimen is often chosen as patients prefer to avoid continuous infusion.

Despite the advantages of CapeOX therapy, guidelines for the management of patients with mCRC published by the European Society for Medical Oncology (ESMO) discourage the use of therapies combining capecitabine with an EGFR antibody [11]. These guidelines were based on evidence showing that treatment with cetuximab + CapeOX has no beneficial effects in these patients [12]. In contrast, a phase II clinical trial we conducted found that combining cetuximab with the oxaliplatin-based FOLFOX or CapeOX treatments yielded positive response rates (RR) of $64.9 \%$ (24/37 patients) and $72.0 \%$ (18/25 patients), respectively, in patients with KRAS and BRAF wild-type mCRCs [13]. Our subsequent phase II trial focusing on a combined CapeOX plus cetuximab treatment in patients with KRAS exon 2 codon $12 / 13$ wild-type mCRC had only a 50\% RR (95\% CI, 33.8-66.2\%), even though the safety profile was acceptable [14].

It is possible that the efficacy of combined CapeOX plus cetuximab treatment as a first-line therapy for mCRCs may indeed be inferior, as suggested by a large phase III clinical trial [12]. However, we believe that the low response rate in our second phase II trial [14] may be due to the coincideental recruitment of patients with $\mathrm{mCRC}$ harboring mutations that we had not identified. Thus, in the present study, we extended the mutation status of
mCRCs to include RAS, BRAF, and PIK3CA mutations. Using this new information, we re-analyzed the clinical efficacy data for CapeOX plus cetuximab treatment from our previous phase II clinical trials.

\section{Methods}

\section{Patients}

The clinical data from two phase II clinical trials FLEET and FLEET2 [13, 14] - were re-analyzed taking into consideration the details of relevant gene mutation status. Patient inclusion criteria and treatment protocols have been described previously $[13,14]$. Briefly, the FLEET study recruited patients with KRAS exon 2 codons 12/13, and exon 3 codon 61 wild-type and BRAF (V600E) wild-type mCRCs. The patients received either FOLFOX or CapeOX as the backbone of their treatment. The FLEET2 study recruited patients with KRAS exon 2 codons 12/13 wild-type $\mathrm{mCRC}$, and they received CapeOX with weekly or biweekly doses of cetuximab.

The choice between FOLFOX or CapeOX, and weekly or biweekly cetuximab administration depended on the preference of the patients and the participating investigators who provided the chemotherapy. Consequently, three different treatment regimens were allowed: FOLFOX + biweekly cetuximab (FOLFOX + cetuximab group), CapeOX + biweekly cetuximab, and CapeOX + weekly cetuximab. As the efficacy and safety of biweekly cetuximab therapy is comparable to weekly cetuximab therapy [15], we combined these two cetuximab therapies into one group, the CapeOX + cetuximab group, for analysis. This study was approved by the institutional review board of Yamaguchi University School of Medicine (Approval number: H28-171), and the institutional review board of Aichi Medical University (Approval number: 2017-H325). The study was performed in accordance with the Declaration of Helsinki.

\section{Detection of mutation status}

Gene mutation status was evaluated using the tissue samples obtained when patients were recruited. Extra tissue samples were not collected and additional biopsies were not performed for the current study. DNA was extracted from formalin-fixed, paraffinembedded tumor tissues [16]. KRAS mutations in codons 12, 13, and 61 [14], and BRAF and PIK3CA mutations were detected by direct sequencing as previously described [17-19]. Extended RAS (KRAS and NRAS) mutations were evaluated using the RASKET KIT [20]. Additionally, microsatellite instability (MSI) was analyzed by using the MSI Analysis System, Version 1.2 according to the manufacturer's instructions (Promega, Madison, WI). 


\section{Endpoints}

The primary endpoint for this analysis was the RR because the previous two studies primarily evaluated the RR of the treatments. The maximum changes in tumor size from baseline in patients with RAS/BRAF/PIK3CA wild-type mCRCs who were treated with different regimens, in patients with mutant-type mCRCs, and between patients with wild-type and mutant-type mCRCs were secondary endpoints. Comparisons of RR between the different regimens and tumor mutation status were also set as main secondary endpoints.

\section{Statistical analysis}

Patient characteristics are presented as the number of patients (n) followed by percentages or median values with the range in parentheses. Statistical comparisons were conducted using the chi-square and Kruskal-Wallis tests. Proportions and corresponding $95 \%$ confidence intervals (CI) were

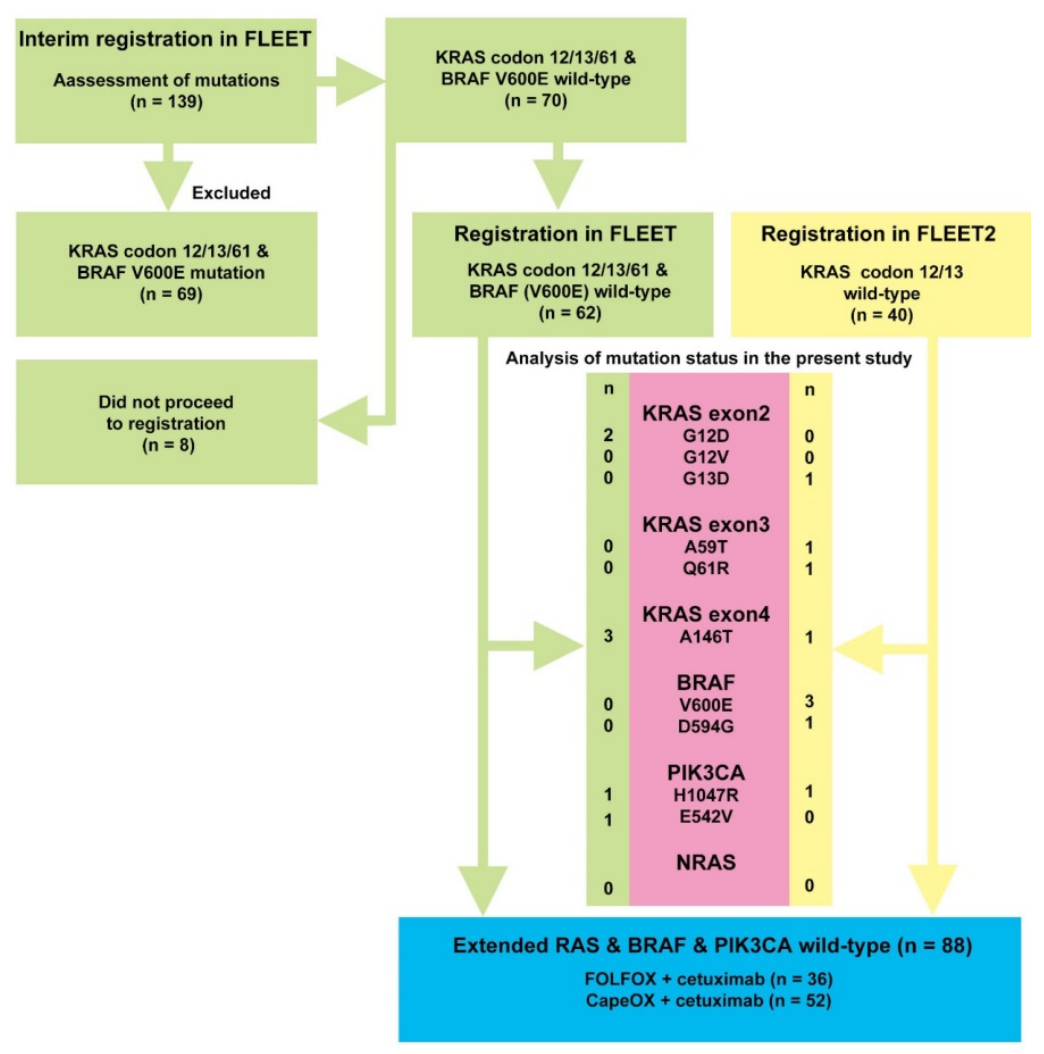

Figure 1. Flow diagram depicting the selection of patients with expanded RAS/BRAF/PIK3CA wild-type mCRCs and patients with $\mathrm{mCRCs}$ with any mutation. A total of 139 patients were recruited at interim registration for the FLEET study. Of these, 69 patients with KRAS exon 2 codons 12/13, and exon 3 codon 61 mutations and the BRAF V600E mutation were excluded. Of the remaining 70 patients, 62 participated in the FLEET study. Clinical course data were available for this study. A total of 40 patients with KRAS exon 2 codons 12/13 wild-type $\mathrm{mCRC}$ were registered in the FLEET2 study. Relevant clinical data were also available for this study. The mutation status of RAS/BRAF/PIK3CA was analyzed in 102 eligible patients recruited for the FLEET and FLEET2 studies. The numbers next to the gene mutation refer to the number of patients with that particular mutation. It should be noted that one patient had both A146T and H1047R mutations, and another patient had D594G and H1047R mutations, simultaneously. Consequently, 88 patients with extended RAS wild-type, BRAF wild-type, and PIK3CA wild-type $\mathrm{mCRCs}$ were identified. calculated for RR and disease control rate (DCR). Maximum changes in tumor size from baseline were compared using the Kruskal-Wallis test. Median overall survival (OS) and progression-free survival (PFS) were calculated using the Kaplan-Meier method. Statistical analyses were performed using SAS version 9.4 (SAS Institute Inc., Cary, NC, USA). In this study, $P<0.05$ was considered significant.

\section{Results}

\section{Patient characteristics}

Of the 102 patients whose tumor mutation status was analyzed, 88 patients had wild-type tumors (Figure 1). The mutation status analyses performed in the present study identified 16 new mutations in 14 12/ 13 were excluded by direct sequencing in the FLEET and FLEET2 studies, re-analysis identified three patients with mutations in KRAS exon 2 codons 12 and 13 (G12D and G13D, respectively). Another six mutations were also identified in KRAS exons 3 and 4 , and PIK3CA mutations were identified in three cases. Four patients with BRAF mutations (V600E), who were excluded in the FLEET trial, were included in the present study. NRAS mutations were not identified in the present study.

Of the 88 patients with extended RAS/BRAF/ PIK3CA wild-type mCRCs, 36 patients received FOLFOX + cetuximab treatment and 52 patients received CapeOX + cetuximab treatment (Table 1). Overall, the median age of patients with extended RAS/BRAF/PIK3CA wild-type mCRC was 66.5 years (range, 33-85 years). Most patients had an Eastern Cooperative Oncology Group Performance Status (ECOG PS) of 0 and the colon was the primary tumor site in 61 patients. The liver was the most common site of metastasis followed by the lymph nodes and lungs. Background differences were found in gender and the prevalence of distant lymph node metastasis. Fewer female patients were found in patients with RAS/BRAF/PIK3CA wild-type mCRCs receiving CapeOX + cetuximab treatment compared to the other two groups. No distant lymph node involvement was detected at registration among the patients with mutant-type mCRCs. In addition, ECOG PS tended to be poorer in patients with mutant-type mCRCs. 


\section{Clinical efficacy}

Overall, the oxaliplatin-based doublet and cetuximab treatment yielded an RR of $60.8 \%$ (95\% CI, $50.6-70.3 \%$ ) and DCF of $88.2 \%$ (95\% CI, 80.4-93.8\%) (Table 2). As the primary endpoint, the RR of CapeOX + cetuximab treatment in patients with extended RAS/BRAF/PIK3CA wild-type mCRCs was $61.5 \%$ (95\% CI, 47.0-74.7\%), while that for FOLFOX + cetuximab was $66.7 \%$ (95\% CI, 49-81.4\%). Meanwhile, for tumors with any mutation had an RR of $42.9 \%$ (95\% CI, 17.7-71.1\%). There was no significant difference between the RRs of the three groups $(P=0.298)$.

\section{Maximum tumor size changes from the baseline}

A waterfall plot revealed a marked reduction in tumor diameter following treatment (Figure 2). Overall, the median maximum tumor size change from baseline was $-52.2 \%$ (95\% CI, -100 to $71.3 \%)$. The maximum tumor size change among patients with wild-type mCRC treated with CapeOX + cetuximab was $-52.6 \%(95 \% \mathrm{CI},-100$ to $71.3 \%)$ while that for patients treated with FOLFOX + cetuximab was $-63.2 \%$ (95\% CI, -100 to $70.2 \%$ ). As observed in Figure 2, patients treated with FOLFOX+ cetuximab accumulated in the left-hand side of waterfall plot, while the majority of patients treated with CapeOX + cetuximab were found in the middle of the plot. The maximum tumor size change from baseline in patients with mutant-type mCRC was $-27.3 \%$ (95\% CI, -100 to $46.2 \%)$, which was significantly smaller than the oxaliplatin-based doublet and cetuximab treatment

changes measured in patients with wild-type mCRC $(P=0.035)$.

\section{Detection of MSI-high patients}

MSI was identified in four cases: there were two cases among patients with wild-type $\mathrm{mCRC}$, and two cases among patients with gene mutations in the tumors. A complete response was observed in one case, and stable disease was observed in three cases (Table 3), yielding an RR of $25 \%$.

Table 1. Characteristics of patients with RAS/BRAF/PIK3CA wild-type or mutant-type advanced/metastatic CRC

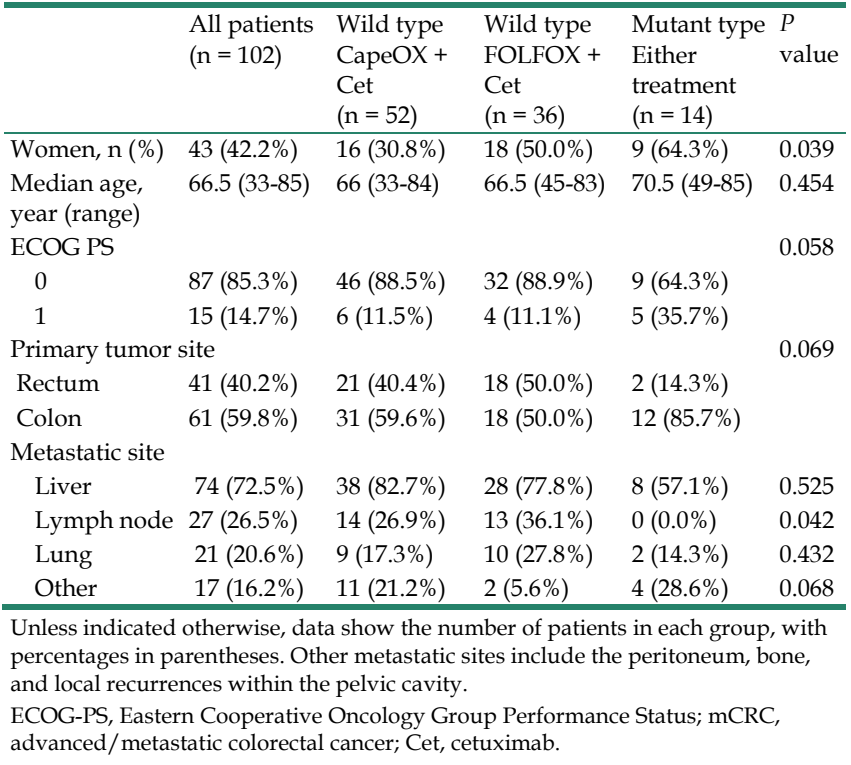

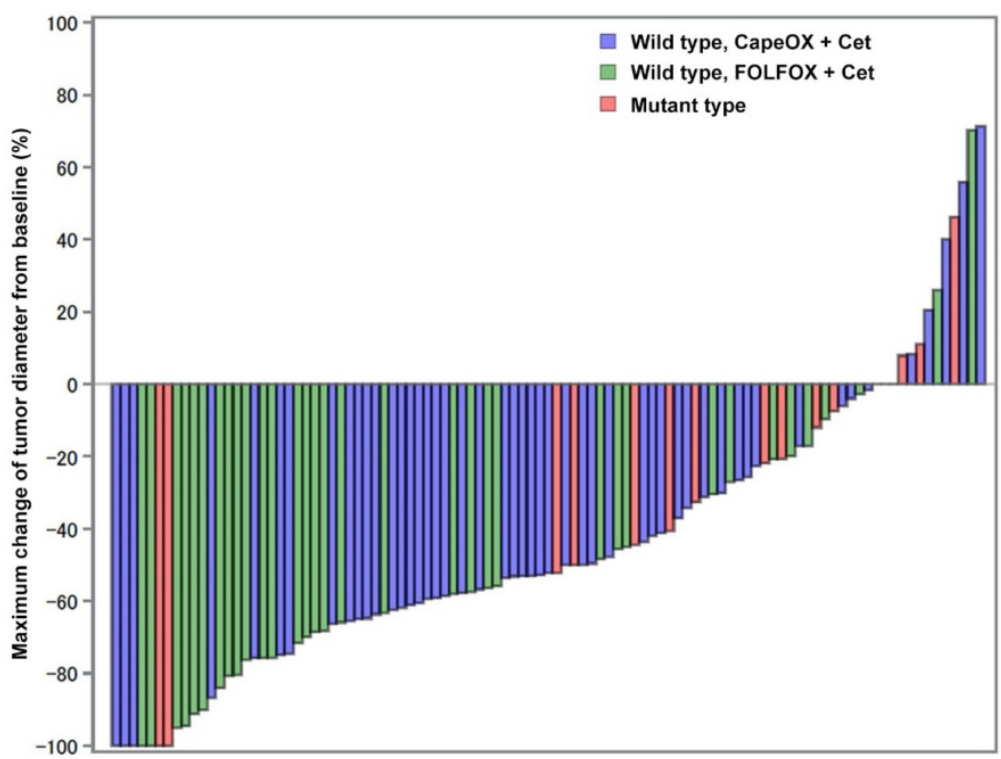

Figure 2. Maximum changes of tumor diameters from baseline. Tumor shrinkage was significantly larger in patients with RAS/BRAF/PIK3CA wild-type mCRCs (blue and green bars) than in patients with any mutation (red bars). Patients treated with FOLFOX + cetuximab appeared more frequently on the left-hand side of this plot suggesting an excellent response to the treatment. Patients treated with CapeOX and cetuximab mainly occupied the middle part of this plot. Patients with any mutation mainly accumulated on the right-hand side of the plot. Despite this, two patients with mutant-type mCRCs still had quite a good response toward combination therapy. 
Table 2. Efficacy of treatment with CapeOX + Cetuximab or FOLFOX + Cetuximab in patients with RAS/BRAF/PIK3CA wild-type or mutant-type advanced/metastatic CRC

\begin{tabular}{|c|c|c|c|c|c|}
\hline & $\begin{array}{l}\text { All patients } \\
(\mathrm{n}=102)\end{array}$ & $\begin{array}{l}\text { Wild type } \\
\text { CapeOX }+ \\
\text { Cet } \\
(n=52)\end{array}$ & $\begin{array}{l}\text { Wild Type } \\
\text { FOLFOX + } \\
\text { Cet } \\
(n=36)\end{array}$ & $\begin{array}{l}\text { Mutant type } \\
\text { Either } \\
\text { treatment } \\
(\mathrm{n}=14)\end{array}$ & $\begin{array}{l}\mathrm{P} \\
\text { value }\end{array}$ \\
\hline \multicolumn{6}{|c|}{ Tumor response } \\
\hline CR & 4 & 1 & 2 & 1 & \\
\hline PR & 58 & 31 & 22 & 5 & \\
\hline SD & 28 & 13 & 8 & 7 & \\
\hline PD & 8 & 5 & 2 & 1 & \\
\hline NE & 4 & 2 & 2 & 0 & \\
\hline RR (\%) & $\begin{array}{l}60.8 \% \\
(50.6-70.3)\end{array}$ & $\begin{array}{l}61.5 \% \\
(47.0-74.7)\end{array}$ & $\begin{array}{l}66.7 \% \\
(49.0-81.4)\end{array}$ & $\begin{array}{l}42.9 \% \\
(17.7-71.1)\end{array}$ & 0.298 \\
\hline DCR $(\%)$ & $\begin{array}{l}88.2 \% \\
(80.4-93.8)\end{array}$ & $\begin{array}{l}86.5 \% \\
(74.2-94.4)\end{array}$ & $\begin{array}{l}88.9 \% \\
(73.9-96.9)\end{array}$ & $\begin{array}{l}92.9 \\
(66.1-99.8)\end{array}$ & 0.800 \\
\hline $\begin{array}{l}\text { Median DpR } \\
(\%)\end{array}$ & $\begin{array}{l}-52.2 \% \\
(-100,71.3)\end{array}$ & $\begin{array}{l}-52.6 \% \\
(-100,71.3)\end{array}$ & $\begin{array}{l}-63.2 \% \\
(-100,70.2)\end{array}$ & $\begin{array}{l}-27.3 \\
(-100,46.2)\end{array}$ & 0.035 \\
\hline $\begin{array}{l}\text { Median PFS } \\
\text { (months) }\end{array}$ & $\begin{array}{l}10.9 \\
(9.0-12.9)\end{array}$ & $\begin{array}{l}10.4 \\
(8.8-13.2)\end{array}$ & $\begin{array}{l}11.1 \\
(7.7-15.5)\end{array}$ & $\begin{array}{l}12.0 \\
(4.9-\mathrm{NA})\end{array}$ & 0.685 \\
\hline $\begin{array}{l}\text { Median OS } \\
\text { (months) }\end{array}$ & $\begin{array}{l}34.0 \\
(26.8-43.9)\end{array}$ & $\begin{array}{l}31.8 \\
(16.7-\mathrm{NA})\end{array}$ & $\begin{array}{l}35.1 \\
(23.7-43.9)\end{array}$ & $\begin{array}{l}\text { NA } \\
\text { (NA-NA) }\end{array}$ & 0.417 \\
\hline
\end{tabular}

Data are provided as the number of patients in each group or as median values with $95 \%$ confidence intervals in parentheses.

$\mathrm{CR}$, complete response; $\mathrm{PR}$, partial response; $\mathrm{SD}$, stable disease; $\mathrm{PD}$, progressive disease; NE, not evaluated; RR, response rate; $D C R$, disease control rate; $D p R$, depth of response; PFS, progression-free survival; OS, overall survival; NA, not available; Cet, cetuximab.

Table 3. Efficacy of treatment with the cetuximab + oxaliplatin backbone regimen in patients with $\mathrm{MSI}$-high advanced/metastatic CRC

\begin{tabular}{ll}
\hline & $\begin{array}{l}\text { MSI high } \\
(\mathrm{n}=4)\end{array}$ \\
\hline Tumor response & \\
CR & 1 \\
PR & 0 \\
SD & 3 \\
PD & 0 \\
NE & 0 \\
RR (\%) & $25.0(0.0-80.6)$ \\
DCR (\%) & $100.0(39.8-100.0)$ \\
Median DpR (\%) & $-25.1(-100,7.9)$ \\
Median PFS (months) & $13.7(9.0-13.7)$ \\
Median OS (months) & $21.8(16.7-26.8)$ \\
\hline
\end{tabular}

$\mathrm{CR}$, complete response; $\mathrm{PR}$, partial response; $\mathrm{SD}$, stable disease; $\mathrm{PD}$, progressive disease; NE, not evaluated; RR, response rate; $\mathrm{DCR}$, disease control rate; $\mathrm{DpR}$ depth of response; PFS, progression-free survival; OS, overall survival; MSI, microsatellite instability

\section{Discussion}

The present study highlights two important messages concerning the treatment of mCRC. The first message is that CapeOX plus cetuximab is not necessarily a detrimental combination when used as a first-line treatment for patients who have extended RAS/BRAF/PIK3CA wild-type mCRCs. Previously, a clinical study known as the COIN trial reported that adding cetuximab to CapeOX therapy had no beneficial effect on the overall survival of mCRC patients [12]. The COIN trial results were reflected in the 2016 edition of the ESMO guidelines for managing patients with mCRC [11]. However, we believe that adjustments to the COIN trial protocol may have masked the clinical benefits of CapeOX treatment. The dose of capecitabine in the CapeOX plus cetuximab arm of the COIN trial was reduced following observations of excess toxicity after the majority of patients had been recruited [21]. Investigators realized that the dose of oxaliplatin was actually less intensive before the capecitabine dose was adjusted; therefore, the altered concentrations of the treatment components might have obscured any benefits associated with the CapeOX plus cetuximab treatment [13]. In addition, the treatment efficacy might have been further undermined because fewer patients in the CapeOX plus cetuximab group received second-line treatment [12].

Pharmacokinetic studies provide further support for combined CapeOX plus cetuximab treatment. Rachar and colleagues demonstrated that the plasma concentrations of capecitabine and its metabolites were not affected even when it was administered with cetuximab [22]. It is also unlikely that co-administration of oxaliplatin, capecitabine, and cetuximab alters the plasma concentration of any of these three agents because no metabolic interactions between these agents have been described [23]. Taken together with our current findings, these pharmacokinetic data plus our concerns about the COIN study, and the lack of other relevant studies demonstrating inferiority of CapeOX plus cetuximab compared to FOLFOX plus cetuximab lead us to suggest that CapeOX plus cetuximab is a potential candidate for use as an active first-line treatment for patients with extended RAS/BRAF/PIK3CA wild-type mCRC. The convenience of this regimen, which has the advantage of not needing infusion catheter placement, further strengthens our claim.

The second message is that the maximum tumor shrinkage observed in patients treated with CapeOX plus cetuximab was smaller than that observed in patients treated with FOLFOX plus cetuximab. In the past, surgical intervention for unresectable hepatic metastasis was an uncommon clinical practice. However, the emergence of bevacizumab and cetuximab in addition to cytotoxic doublets improved conversion rates, and eventually improved overall survival rates [24, 25]. These results suggest that optimal chemotherapy regimens should be provided as first-line treatments, during which time the tumor size must be frequently assessed to gauge whether it is ready for resection [11]. In this context, our results may suggest that CapeOX plus cetuximab treatment is not a suitable strategy for patients with liver-limited unresectable metastases in which significant tumor shrinkage might allow curative resection. However, we should note that several patients receiving 
CapeOX plus cetuximab still showed significant tumor shrinkage, as shown in Figure 2. Further studies are required to examine whether the differences in tumor shrinkage observed in the present study result in altered conversion rates.

According to studies from Western countries, the prevalence of the BRAF mutation is approximately $9 \%$ $[7,26]$. In contrast, we identified the BRAF mutation in $2.8 \%(4 / 139)$ of Japanese patients registered in the FLEET study and in 10\% (4/40) of patients registered in the FLEET2 study. Similarly, a retrospective analysis of Japanese patients identified the BRAF mutation in $5.4 \%$ of Japanese patients [27]. These results suggest that BRAF mutations are less common in Japanese $\mathrm{mCRC}$ patients than in Western patients. In the present study, the clinical course of only four patients with BRAF mutation mCRC were available: two patients had a partial response during the treatment protocol, whereas the disease remained stable in the other two patients.

High levels of microsatellite instability (MSI-H) are found in approximately $5 \%$ of patients with colorectal cancer. MSI-H are associated with poor prognosis, which may be attributable to co-existing BRAF mutations [27]. In the present study, three out of four patients with MSI-H did not have a BRAF mutation. Two of these three patients had a lukewarm response (stable disease) to treatment, with a maximum tumor size change of $-20 \%$ and $-30.1 \%$, respectively. These results may suggest that MSI-H is a predictor of poor response to treatment regardless of BRAF mutation status. However, this is purely speculative due to the small number of patients.

The limitations of the present study include the small number of patients, the lack of information concerning the original tumor site (left-right), missing information regarding the interval between curative resection of primary tumors to detection of recurrence, and/or the lack of clinical information on patients with BRAF mutation $\mathrm{mCRC}$ recruited in the FLEET study. These factors may potentially influence the clinical course of the treatment $[28,29]$, and alter the interpretation of the results. Especially, a retrospective study focusing on the location of the primary tumor revealed that patients who had a left-sided tumor had a better prognosis than those who had a right-sided tumor, showing a clear clinical benefit of cetuximab treatment in patients with left-sided tumors [30]. However, few studies have addressed this idea, and further research is needed. An analysis using data from the 153 patients treated with cetuximab-containing chemotherapy for metastatic colorectal cancer did not show a clear difference in PFS between right- and left-sided tumors [31]. The authors noted that effects of tumor location on clinical outcomes might have been masked in this analysis for two reasons: lack of information regarding oncogene mutations and/or epigenetic alteration, and the inclusion of different lines of treatments. Therefore, including sidedness of the primary tumor as a variable in the present study would have provided further insight into the relationships between cetuximab efficacy, gene mutations, and primary tumor sidedness.

\section{Conclusions}

In conclusion, first-line treatment with CapeOX plus cetuximab for patients with extended RAS/BRAF/PIK3CA wild-type mCRCs showed an appropriate response rate. Due to the convenience of this treatment regimen, CapeOX plus cetuximab should remain a treatment option for patients with mCRC.

\section{Acknowledgements}

This study was supported by the non-profit organization Epidemiological and Clinical Research Organization (ECRIN). This work was achieved thanks to all of the patients and investigators involved.

\section{Authors' contributions}

SI, HM, SH, KO, JS, HM, and NN contributed to the study conception and design. NO, YS, TY, NS, $\mathrm{HN}$, JS, HM, and NN significantly contributed to data acquisition and laboratory tests. SI and $\mathrm{KO}$ contributed to data analysis and all authors contributed to its interpretation. SI, HM, JS, HM, and NN contributed to manuscript preparation, editing, and graphic depiction. All authors reviewed and approved the final version of the manuscript.

\section{Disclosure}

JS has received honoraria from Tsumura, Chugai Pharmaceutical, and consulting fee from Takeda Pharmaceutical. KO has received honoraria (lecture and/or manuscript fee) from Takeda Pharmaceutical Company Ltd., Bristol-Myers Squibb Company Ltd., Ono Pharmaceutical Co. Ltd., and Chugai Pharmaceutical Co. Ltd. Shoichi Hazama and Hiroaki Nagano received research funding from NEC Corporation, Toyo Kohan Corporation, and Merck Serono Co., Ltd. HM has received honoraria from Chugai Pharmaceutical, Takeda Pharmaceutical, and Taiho Pharmaceutical. NN has received honoraria from Takeda Pharmaceutical Company Ltd. Other co-authors declare no conflict of interest.

\section{References}

1. Baselga J. Why the epidermal growth factor receptor? The rationale for cancer therapy. Oncologist. 2002; 7 Suppl 4: 2-8. 
2. Jonker DJ, O'Callaghan CJ, Karapetis CS, et al. Cetuximab for the treatment of colorectal cancer. N Engl J Med. 2007; 357: 2040-8.

3. Bokemeyer C, Bondarenko I, Makhson A, et al. Fluorouracil, leucovorin, and oxaliplatin with and without cetuximab in the first-line treatment of metastatic colorectal cancer. J Clin Oncol. 2009; 27: 663-71.

4. Van Cutsem E, Köhne CH, Hitre E, et al. Cetuximab and chemotherapy as initial treatment for metastatic colorectal cancer. N Engl J Med. 2009; 360: 1408-17.

5. Karapetis CS, Khambata-Ford S, Jonker DJ, et al. K-ras mutations and benefit from cetuximab in advanced colorectal cancer. N Engl J Med. 2008; 359: 1757-65.

6. Stintzing S, Miller-Phillips L, Modest DP, et al. Impact of BRAF and RAS mutations on first-line efficacy of FOLFIRI plus cetuximab versus FOLFIRI plus bevacizumab: analysis of the FIRE-3 (AIO KRK-0306) study. Eur J Cancer. 2017; 79: 50-60

7. Pietrantonio F, Petrelli F, Coinu A, et al. Predictive role of BRAF mutations in patients with advanced colorectal cancer receiving cetuximab and panitumumab: a meta-analysis. Eur J Cancer. 2015; 51: 587-94.

8. De Roock W, Claes B, Bernasconi D, et al. Effects of KRAS, BRAF, NRAS, and PIK3CA mutations on the efficacy of cetuximab plus chemotherapy in chemotherapy-refractory metastatic colorectal cancer: a retrospective consortium analysis. Lancet Oncol. 2010; 11: 753-62.

9. Chu E, Haller D, Cartwright T, et al. Epidemiology and natural history of central venous access device use and infusion pump function in the NO16966 trial. Br J Cancer. 2014; 110: 1438-45.

10. Cassidy J, Clarke S, Díaz-Rubio E, et al. Randomized phase III study of capecitabine plus oxaliplatin compared with fluorouracil/folinic acid plus oxaliplatin as first-line therapy for metastatic colorectal cancer. J Clin Oncol. 2008; 26: 2006-12

11. Van Cutsem E, Cervantes A, Adam R, et al. ESMO consensus guidelines for the management of patients with metastatic colorectal cancer. Ann Oncol. 2016; 27: 1386-422.

12. Maughan TS, Adams RA, Smith CG, et al. Addition of cetuximab to oxaliplatin-based first-line combination chemotherapy for treatment of advanced colorectal cancer: results of the randomised phase 3 MRC COIN trial. Lancet. 2011; 377: 2103-14.

13. Soda H, Maeda H, Hasegawa J, et al. Multicenter Phase II study of FOLFOX or biweekly XELOX and Erbitux (cetuximab) as first-line therapy in patient with wild-type KRAS/BRAF metastatic colorectal cancer: The FLEET study. BMC Cancer. 2015; 15: 695.

14. Hazama S, Maeda H, Iwamoto S, et al. A Phase II Study of XELOX and Cetuximab as First-Line Therapy in Patients With KRAS Wild Type Metastatic Colorectal Cancer (FLEET2 Study). Clin Colorectal Cancer. 2016; 15: 329-36.

15. Fernandez-Plana J, Pericay $C$, Quintero $G$, et al. Biweekly cetuximab in combination with FOLFOX-4 in the first-line treatment of wild-type KRAS metastatic colorectal cancer: final results of a phase II, open-label, clinical trial (OPTIMIX-ACROSS Study). BMC Cancer. 2014; 14: 865.

16. Okayama N, Nishioka M, Hazama S, et al. The importance of evaluation of DNA amplificability in KRAS mutation testing with dideoxy sequencing using formalin-fixed and paraffin-embedded colorectal cancer tissues. Jpn J Clin Oncol. 2011; 41: 165-71.

17. Iwamoto S, Hazama S, Kato T, et al. Multicenter phase II study of second-line cetuximab plus folinic acid/5-fluorouracil/irinotecan (FOLFIRI) in KRAS wild-type metastatic colorectal cancer: the FLIER study. Anticancer Res. 2014; 34: 1967-73.

18. Kim YH, Kakar S, Cun L,et al. Distinct CpG island methylation profiles and BRAF mutation status in serrated and adenomatous colorectal polyps. Int J Cancer. 2008; 123 : 2587-93.

19. Jhawer M, Goel S, Wilson AJ, et al. PIK3CA mutation/PTEN expression status predicts response of colon cancer cells to the epidermal growth factor receptor inhibitor cetuximab. Cancer Res. 2008; 68: 1953-61.

20. Yoshino T, Muro K, Yamaguchi K, et al. Clinical Validation of a Multiplex Kit for RAS Mutations in Colorectal Cancer: Results of the RASKET (RAS KEy Testing) Prospective, Multicenter Study. EBioMedicine. 2015; 2: 317-23.

21. Adams RA, Meade AM, Madi A, et al. Toxicity associated with combination oxaliplatin plus fluoropyrimidine with or without cetuximab in the MRC COIN trial experience. Br J Cancer. 2009; 100: 251-8.

22. Rachar V, Czejka M, Kitzmueller M, et al. Assessment of Pharmacokinetic Interaction Between Capecitabine and Cetuximab in Metastatic Colorectal Cancer Patients. Anticancer Res. 2016; 36: 4715-23.

23. Farkouh A, Schueller I, Scheithauer $W$, et al. Plasma disposition of capecitabine (CCB) and its metabolites 5'-deoxy-5-fluorocytidine (5'-DFCR) and 5'-deoxy-5-fluorouracil (5'-DFUR) with two different capecitabine/oxaliplatin dosage regimens. Int J Clin Pharmacol Ther. 2010; 48: 487-8.

24. Folprecht G, Gruenberger T, Bechstein WO, et al. Tumour response and secondary resectability of colorectal liver metastases following neoadjuvant chemotherapy with cetuximab: the CELIM randomised phase 2 trial. Lancet Oncol. 2010; 11: 38-47.

25. Folprecht $G$, Gruenberger T, Bechstein W, et al. Survival of patients with initially unresectable colorectal liver metastases treated with FOLFOX/cetuximab or FOLFIRI/cetuximab in a multidisciplinary concept (CELIM study). Ann Oncol. 2014; 25: 1018-25.
26. Venderbosch S, Nagtegaal ID, Maughan TS, et al. Mismatch repair status and BRAF mutation status in metastatic colorectal cancer patients: a pooled analysis of the CAIRO, CAIRO2, COIN, and FOCUS studies. Clin Cancer Res. 2014; 20: 5322-30.

27. Kawazoe A, Shitara K, Fukuoka S, et al. A retrospective observational study of clinicopathological features of KRAS, NRAS, BRAF and PIK3CA mutations in Japanese patients with metastatic colorectal cancer. BMC Cancer. 2015; 15: 258

28. O'Connell MJ, Campbell ME, Goldberg RM, et al. Survival following recurrence in stage II and III colon cancer: findings from the ACCENT data set. J Clin Oncol. 2008; 26: 2336-41.

29. Brulé SY, Jonker DJ, Karapetis CS, et al. Location of colon cancer (right-sided versus left-sided) as a prognostic factor and a predictor of benefit from cetuximab in NCIC CO.17. Eur J Cancer. 2015; 51: 1405-14.

30. Tejpar S, Stintzing S, Ciardiello F, et al. Prognostic and Predictive Relevance of Primary Tumor Location in Patients With RAS Wild-Type Metastatic Colorectal Cancer: Retrospective Analyses of the CRYSTAL and FIRE-3 Trials. JAMA Oncol. 2016. [Epub ahead of print]

31. Kim ST, Lee SJ, Lee J, et al. The Impact of Microsatellite Instability Status and Sidedness of the Primary Tumor on the Effect of Cetuximab-Containing Chemotherapy in Patients with Metastatic Colorectal Cancer. J Cancer. 2017 8: 2809-2815. 